\title{
Population based ultrasonographic screening of abdominal aortic aneurysms
}

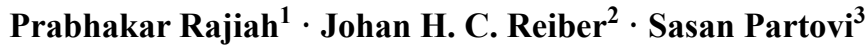

Received: 22 July 2016 / Accepted: 26 July 2016 / Published online: 27 July 2016

(C) Springer Science+Business Media Dordrecht 2016

\begin{abstract}
Abdominal Aortic Aneurysm (AAA), defined as an infrarenal aortic diameter $>3.0 \mathrm{~cm}$ is not infrequently seen in the older population, especially in male smokers. The overall prevalence of AAA in men $>50$ years is $3.9-7.2 \%(6-7 \%$ in smokers, $2 \%$ in non-smokers) whereas in women the overall prevalence is $1.0-1.3 \%(0.8-2.0 \%$ in smokers, $0.03-0.6 \%$ in non-smokers) [1]. AAA is also associated with a positive family history and thoracic aortic aneurysms [2]. Although AAA is usually asymptomatic, the first presentation may be a rupture, the probability of which increases with size of the aneurysm sac $(6.5-11 \%$ for $5-5.9 \mathrm{~cm}, 1 \%$ for $4-4.9 \mathrm{~cm}, 0 \%$ for 3.0-3.9 cm) $[1,3]$ and it is three times more common in females at comparable diameters [4]. Rupture has a high mortality rate (75-90\%), with the majority (59-83\%) passing before reaching the hospital and the operative mortality is high as well (40\%) [1]. To avoid the risk of rupture, AAA is electively repaired, either using an endovascular (EVAR) or open approach, when it is $>5.5 \mathrm{~cm}$ or grows $>1.0 \mathrm{~cm} /$ year $[5,6]$. Hence, an early diagnosis of AAA is imperative to positively impact patient outcome. Physical examination has been shown to have low sensitivity (39-68\%) and specificity $(75 \%)[5,6]$. CT imaging has high sensitivity $(90 \%)$ and specificity $(91 \%)$, but associated with higher cost and
\end{abstract}

Prabhakar Rajiah

radprabhakar@gmail.com

1 Department of Radiology, Cardiothoracic Imaging, UT Southwestern Medical Center, 5323 Harry Hines Boulevard, Dallas, TX 75390, USA

2 Department of Radiology, Leiden University Medical Center, Leiden, The Netherlands

3 Department of Radiology, University Hospitals Case Medical Center, Cleveland, OH, USA radiation exposure. Non-contrast CT may be utilized when ultrasound (US) is not suitable and CTA is also useful in pre-procedural planning in delineating the type, size, extent and location of aneurysm as well as branch vessel involvement [7]. MRI is an attractive alternative modality for evaluating AAA as well as other vascular systems [8], but it is not widely available, expensive, and time-consuming and requires higher technical expertise. MRA can be used for pre-procedural planning if CT cannot be performed [7]. US is non-invasive, widely available, portable, cost effective and has no radiation as well has high sensitivity (94-100\%), specificity (98-100\%) and reproducibility [9] in detection of AAA, making it the ideal screening modality for AAA.

The benefits of AAA screening using a one-time US have been demonstrated by several large randomized controlled trials in UK, Denmark and Western Australia $[1,10]$. AAA-related mortality has been shown to decrease by $40 \%$ at $3-5$ years [11], and $42-66 \%$ at 13 years [12, 13], with an absolute decline in mortality by 1.4 per 1000 men [12]. AAA-mortality at $5-12$ years was shown to be $0-2.4 \%$ [5, 6], with a $42-52 \%$ decrease of AAA related death in those who attended screening $[12,13]$. All-cause mortality was also shown to be slightly decreased by $3 \%$ at $11-15$ years, possibly due to management of other cardiovascular risk factors $[1,12]$. The incidence of rupture was also significantly reduced (Hazard ratio of $0.44-0.57$ ) up to 13 years $[12,13]$. The rate of emergency repair was halved and elective repair doubled upto $13-15$ years $[12,13]$. This robust data led the United States Preventive Services Task Force (USPSTF) to recommend a one-time US AAA screening in male smokers of age 65-75 years. For the same age group, it recommends selective use in male never-smokers and advocates against screening for female never-smokers. No specific recommendation is made for female smokers due to insufficient evidence [1]. In the current issue of 
International Journal of Cardiovascular Imaging, Corrado et al. [4], performed a convincing population based study in Como, Northwest Italy over a study period of slightly above 2 years to evaluate screening US examination of the infrarenal aorta in 1555 asymptomatic individuals (female and male) between 60 and 85 years without a history of AAA. In details, one-time free US along with clinical examination was offered to a large cohort of asymptomatic people in $60-85$ age group, i.e. 1555 people ( $3 \%$ of the entire population) out of 600,000 (51.4\% males, $48.6 \%$ females; age $68.8 \pm 6.8$ years) over a period of 26 months. This population based screening program appears to be the largest AAA screening study performed in Italy. The screening program was successful and the study found an overall AAA prevalence of $1.4 \%$ (equals 22 enrolled subjects), with higher prevalence in males $(2.5 \%$ vs. $0.4 \%, p$ 0.005). In line with previous studies, the univariate analysis revealed male gender, increasing age, history of smoking (including previous and current smoking), hyperlipidemia and history of coronary artery disease as AAA predictors. On multivariate analysis male gender, increasing age and history of smoking (including previous and current smoking) were determined as independent predictors of AAA [4].

AAA screening with US is not without controversies, particularly the risk of overtreatment. Majority of the aneurysms detected with screening are small, with only 0.4 $0.6 \%>5.0 / 5.5 \mathrm{~cm}[5,6]$. No benefit has been shown with elective repair of smaller aneurysms [1]. Also, sub- aneurysmal aortas $(2.5-2.9 \mathrm{~cm})$, accounting for $2 \%$ of population have a $50 \%$ risk of progression to true AAA in 5 years [14]. This often results in long term, potentially life-long surveillance, with not only direct costs of imaging but also potential opportunity costs of foregoing another preventive measure in health systems with scarce resources. However, similar to other imaging interventions, US has been shown to be overall highly cost-effective than no imaging or observation alone [15]. There are conflicting results on quality of life, anxiety and depression associated with long-term screening [1], with some studies showing mild transient reduction of quality of life [14]. There is doubling of AAArelated surgeries at 3-5 years, persisting up to $13-15$ years $[5,6]$. This is not necessarily a disadvantage as these elective surgeries reduce the overall mortality and improve outcomes. However, since these elective surgeries do have risk of perioperative complications and only half of the repaired AAAs after screening would have ruptured without treatment, there is an ethical dilemma of offering screening and thus potentially surgery with risks to asymptomatic people. However, this can be counterbalanced by the fact that the risk of death with elective surgeries is lower in screening population than incidentally discovered AAA [14] and that the mortality from elective procedures is markedly lower than emergency surgeries. Interestingly there is a higher utility of open than endovascular surgeries in women, potentially due to smaller access vessels, unfavorable neck anatomy, as well as a higher post-operative complications and mortality $[5,6]$. Overall elderly female smokers may benefit from US AAA screening. As described above, AAA in elderly female patients is associated with worse outcome including an increased risk for rupture and therefore this population should be included in US AAA screening programs.

The study by Corrado et al. study also illustrates another interesting trend, i.e. an overall decrease in prevalence of AAA in the population, which is concordant with results from other recent studies from UK, New Zealand and Sweden which have shown prevalence from 1.1 to $1.7 \%$ [4-6, 16]. There are several potential reasons for this decline, chief among which is the successful public health effort of smoking cessation [4]. Incidental detection of AAAs by other tests such as CT also decreases the prevalence in screening programs. There has also been an overall decrease in the number of repairs for ruptured aneurysms, which could be partly attributed to successful screening programs, but also possibly contributed by overall decrease in the AAA prevalence. Declining rates of rupture and mortality unrelated to screening have been reported [14]. Advances in endovascular and surgical techniques have also improved outcomes, even for ruptured aneurysms.

Systematic reviews based on the prevalence in early trials have shown a consistent lifetime absolute risk reduction (ARR) from AAA-related death of 7.8 per 10,000 invited for screening and incremental cost effectiveness ratio (ICER) per quality adjusted life year (QALY) gained and life year (LY) gained of $€ 6622$ and $€ 5783$ respectively. Extrapolating this to the current prevalence of $1.7 \%$ results in ARR of 15.1 per 10,000 invited to screening after 13 years followup [14]. This is comparable to ARR for colorectal cancer by fecal occult blood testing (15 per 10,000) and superior to breast cancer by mammography $(5$ per 10,000$)$ [14], validating the relevance of US AAA screening. The cost effectiveness may be potentially decreased by lower prevalence in screening programs due to incidental detection by other tests. However, this is counterbalanced by the increasing longevity of the population in general and improved endovascular and surgical techniques with decreasing perioperative mortality which not only result in improved outcomes but also facilitate more elderly people eligible for surgery, all of which increase the cost effectiveness and life years saved and unchanged low cost per QALY gained [14].

\section{Compliance with ethical standards}

\section{Conflict of interest None.}

Ethical approval This article does not contain any studies with human participants of animals performed by any of the authors. 


\section{References}

1. Final Recommendation Statement: Abdominal Aortic Aneurysm: Screening.U.S.PreventiveServicesTaskForce.June2014.http://www. uspreventiveservicestaskforce.org/Page/Document/Recomm endationStatementFinal/abdominal-aortic-aneurysmscreening

2. DeFreitas MR, Quint LE, Watcharatone K et al (2016) Evaluation of abdominal aortic aneurysms is justified in patients with thoracic aortic aneurysms. Int J Cardiovasc Imaging 32(4):647-653

3. Brown PM, Pattenden R, Vernooy C et al (1996) Selective management of abdominal aortic aneurysms in a prospective measurement program. J VascSurg 23:213-220

4. Corrado G, Durante A, Genchi V et al (2016) Prevalence of previously undiagnosed abdominal aortic aneurysms in the area of Como: the ComoCuore "looking for AAA" ultrasonography screening. Int J Cardiovasc Imaging. doi:10.1007/ s10554-016-0911-3

5. Guirguis-Blake JM, Beil TL, Sun X et al (2014) Primary care screening for abdominal aortic aneurysm: An evidence update for the US preventive Services Task Force. Evidence Synthesis No. 109. AHRQ Publication No. 14-05202-EF-1. Rockville MD, Agency for Healthcare Research and Quality

6. Guirguis-Blake JM, Beil TL, Sun X et al (2014) Ultrasonography screening for abdominal aortic aneurysms a systemic evidence review for the US Preventive services Task Force. Ann Intern Med 160:321-329

7. Desjardins B, Dill KE, Flamm SD et al (2013) ACR appropriateness criteria pulsatile abdominal mass, suspected abdominal aneurysm. Int J Cardiovasc Imaging 29(1):177-183

8. Ruehm SG, Goehde SC, Goyen M (2004) Whole body MR angiography screening. Int J Cardiovasc Imaging 20(6):587-591
9. Chaikof EL, Brewster DC, Dalman RL et al (2009) Society for Vascular Surgery. The care of patients with an abdominal aortic aneurysm: the Society for Vascular Surgery Practice guidelines. J VascSurg 50:S2-S49

10. Ashton HA, Buxton MJ, Day NE et al (2002) The Multicenter Aneurysm Screening Study (MASS) into the effect of abdominal aortic aneurysm screening on mortality in men: a randomized controlled trial. Lancet 360:1531-1539

11. Cosford PA, Leng PC (2007) Screening for abdominal aortic aneurysm. Cochrane Database Syst Rev CD 002945

12. Thompson SG, Ashton HA, Gao L et al (2012) Multicenter Aneurysm Screening Study (MASS) Group. Final follow up of the Multicenter Aneurysm Screening Study (MASS) randomized trial of abdominal aortic aneurysm screening. Br J Surg 99(12):1649-1656

13. Lindholt JS, Sorensen J, Sogaard R et al (2010) Long-term benefit and cost-effectiveness analysis of screening for abdominal aortic aneurysms from a randomized controlled trial. Br J Surg 97(6):826-834

14. Svensjo S, Bjorck M, Wanhainen A (2014) Update on screening for abdominal aortic aneurysm: a topical review. Eur J Vasc Endovasc Surg 48(6):659-667

15. Otero HJ, Rybicki FJ, Greenberg D et al (2010) Cost-effective diagnostic cardiovascular imaging when does it provide good value for money? Int J Cardiovasc Imaging 26(6):605-612

16. Svensjo S, Bjorck M, Gurtelschmid M et al (2011) Low prevalence of abdominal aortic aneurysm among 65 year old Swedish men indicates a change in the epidemiology of the disease. Circulation 124:1118-1123 